\title{
Réformes structurelles et politiques macroéconomiques
}

Structural reforms and macroeconomic policies

\section{Charles Wyplosz}

\section{(2) OpenEdition \\ Journals}

Édition électronique

URL : http://journals.openedition.org/travailemploi/3595

DOI : 10.4000/travailemploi.3595

ISSN : 1775-416X

Éditeur

DARES - Ministère du Travail

Édition imprimée

Date de publication : 5 juin 2009

Pagination : 43-47

ISSN : 0224-4365

Référence électronique

Charles Wyplosz, «Réformes structurelles et politiques macroéconomiques », Travail et Emploi [En ligne], 118 | avril-juin 2009, mis en ligne le 03 décembre 2010, consulté le 30 avril 2019. URL : http:// journals.openedition.org/travailemploi/3595 ; DOI : 10.4000/travailemploi.3595 


\title{
Réformes structurelles et politiques macroéconomiques
}

\author{
Charles Wyplosz (*)
}

\section{Pourquoi évoquer les politiques macroéconomiques?}

Dans son rapport sur l'emploi (OCDE, 2006), l'OCDE tire le bilan d'une décennie de réformes du travail et propose une stratégie pour approfondir et améliorer les résultats acquis. Comme toute réforme structurelle, une Stratégie pour l'emploi est, par définition, une approche qui privilégie le long terme. Or la présomption est que les politiques macroéconomiques ont, au mieux, uniquement des effets à court et moyen terme, mais pas d'effets permanents. A priori, donc, la question peut paraître surprenante. Avant de tirer une conclusion négative, il peut être utile de réexaminer la question. non inflationniste). Ces comouvements sont également visibles sur le graphique de droite, mais ce qui domine ici est le fait que l'évolution de long terme du taux de chômage apparaît comme complètement découplée des fluctuations de court-terme. De même, il n'existe pas de relation de long terme entre croissance et chômage ou emploi.

\section{L'hypothèse d'hystérèse}

Ces résultats sont conformes aux principes habituels. L'exception majeure est l'hypothèse d'hystérèse. Initialement formulée par BLANCHARD et SuMMERs (1986), cette hypothèse évoque la possibilité que des variations temporaires du niveau d'activité peuvent avoir des effets permanents sur l'emploi ou le chômage. Divers mécanismes sont envisageables. Tous imaginent, par exemple, qu'une période de

Figure 1: Activité et chômage
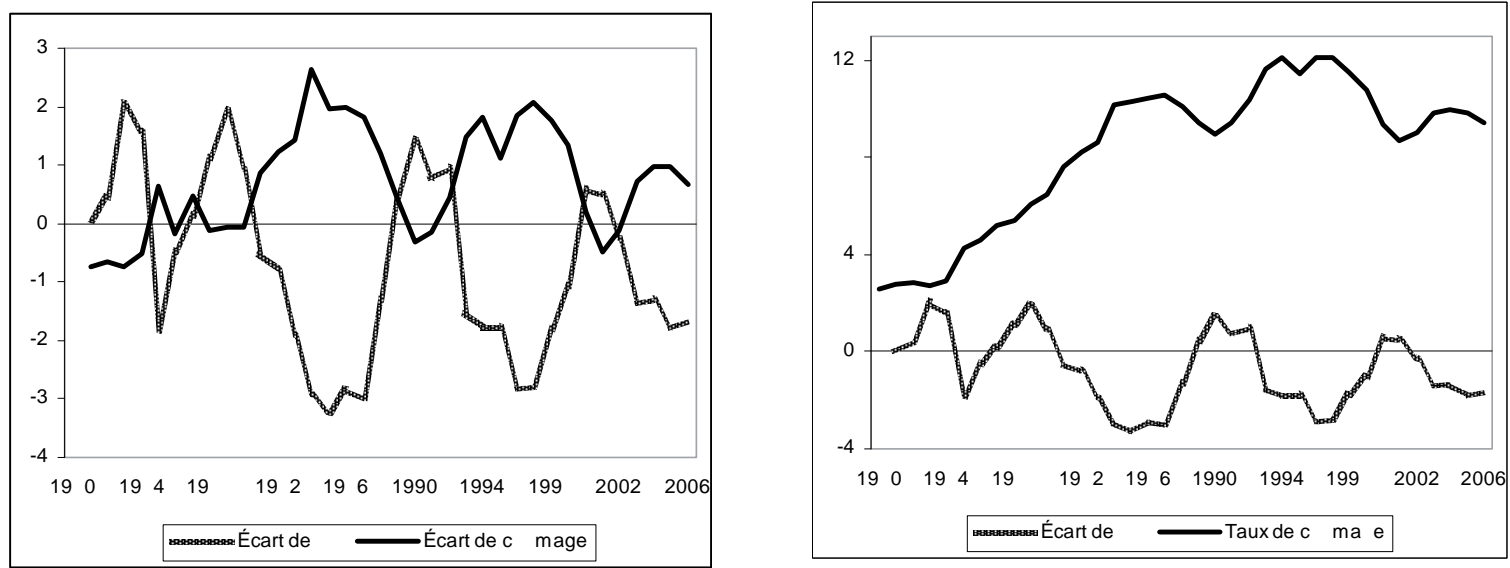

Source: Perspectives économiques, OCDE, 2007.

Note: l'écart de chômage est la différence entre le taux observé et le NAIRU.

Le lien cyclique entre chômage et croissance est, bien sûr, indéniable. La loi d'Okun apparaît clairement dans le premier graphique de la figure 1 qui montre l'évolution de l'écart de PIB (la différence, en pourcentage, entre le PIB réel et son nouveau potentiel) et de l'écart de chômage (la différence entre le taux de chômage et son niveau d'équilibre

* Institut universitaire de hautes études internationales, Genève : charles.wyplosz@graduateinstitue.ch croissance rapide attire dans la population active des personnes qui n'y étaient pas, soit qu'elles soient découragées par de faibles perspectives d'emploi, soit parce que les conditions offertes étaient insuffisamment incitatives. Une fois actives, ces personnes se réinsèrent durablement dans le monde du travail. À l'inverse, une période de croissance faible aurait des effets négatifs durables sur l'emploi.

L'hypothèse d'hystérèse a été l'objet d'études approfondies. Une revue et mise à jour récente de ces travaux par Gustavsson, Magnus et Pär 
ÖSTERHOLM (2006) confirme que l'hypothèse n'est pas, en général, confirmée par les faits. Autrement dit, demander à la politique macroéconomique de «donner un coup de reins» pour accélérer temporairement la croissance ne peut être justifié par l'idée qu'il en résultera un bénéfice durable en matière d'emploi. La politique macroéconomique continue à avoir un rôle à jouer pour gommer les cycles et les fluctuations en matière d'emplois, mais dès lors qu'il s'agit de s'attaquer au problème du sous-emploi, la réponse ne peut qu'être microéconomique, c'est-à-dire structurelle et agissant par le côté de l'offre.

\section{Accompagner l'offre}

Une autre raison parfois évoquée pour justifier un recours à la politique macroéconomique en matière de stratégie d'emploi est qu'il ne sert à rien de stimuler l'emploi si la demande n'est pas au rendez-vous. Les politiques structurelles visent toutes, d'une manière ou d'une autre, à encourager les entreprises à augmenter le contenu en emploi de leurs procédés de production (dans le secteur des services comme dans celui des biens) et à encourager les personnes non-actives ou au chômage à rechercher plus activement des emplois et à les accepter lorsqu'ils se présentent. Ces mesures reposent sur des incitations, mais les incitations ne sont opérantes que si la situation macroéconomique permet une expansion suffisante de l'activité.

Ce raisonnement est incomplet sur le plan théorique. La loi de Say, plus généralement les principes d'équilibre général, suggèrent qu'une augmentation de l'offre crée les conditions d'une augmentation de la demande, à condition que les prix soient flexibles. Bien sûr, les prix - et les salaires - ne sont pas flexibles, surtout à la baisse. On retrouve donc, semble-t-il, le traditionnel cas keynésien et donc un rôle pour la politique macroéconomique. Mais la réponse doit être affinée.

Un premier élément à prendre en compte est que la quasi-totalité des politiques structurelles qui visent à accroître l'emploi passent par une baisse, directe ou induite, des coûts du travail. Les politiques structurelles créent donc les conditions d'une flexibilité à la baisse des coûts du travail. Dans la mesure où les marchés des biens et services sont concurrentiels, on est en droit d'attendre une baisse des prix. Ainsi, cette observation met l'accent sur une des innovations de la nouvelle Stratégie pour l'emploi de l'OCDE: il ne suffit pas de flexibiliser le marché du travail, il est tout aussi essentiel de s'assurer que les marchés des biens et services sont concurrentiels. La réponse est donc en premier lieu structurelle, et non macroéconomique.

Il n'en reste pas moins que l'on ne peut jamais être sûr que l'offre créera la demande nécessaire pour que les politiques structurelles déploient tous les effets attendus. Quoi que l'on fasse, il est probable qu'il restera toujours suffisamment de rigidités des prix pour justifier une politique macroéconomique d'accompagnement. Deux conclusions s'ensuivent. Tout d'abord, la politique macroéconomique garde toute son importance et doit, en effet, soutenir l'activité dès lors qu'elle ne se développe pas en fonction du potentiel de l'économie. Ici, donc, rien de nouveau. Mais, et c'est le second point, en période d'ajustement structurel, il devient important de bien mesurer l'évolution du potentiel. Les indicateurs traditionnels - notamment l'écart de PIB et le taux de chômage d'équilibre - sont peu précis et demandent souvent une longue période d'observation avant d'être réajustés. On se souviendra, par exemple, des débats passionnés lorsque les ÉtatsUnis ont amorcé il y a une dizaine d'années, leur révolution des technologies de l'information. Il semble que les autorités aient correctement perçu les implications de ce changement structurel, mais les débats furent vifs.

La conclusion est donc qu'il n'est pas opportun de procéder par anticipation à une politique macroéconomique expansionniste pour accompagner une politique structurelle; la politique macroéconomique doit simplement jouer son rôle habituel. Doitelle être ajustée à l'avance? Il semble préférable de ne pas s'y essayer. Anticiper les effets positifs de la politique structurelle est hasardeux, car ses effets sont lents à apparaître, et que l'on ignore quand ils le feront. Les expériences dans de nombreux pays suggèrent qu'il faut compter plusieurs années, mais il est en général difficile d'être précis. Ceci dit, il importe de s'attacher à détecter au plus tôt ces effets pour s'assurer que la politique macroéconomique, qui doit contribuer à maintenir le niveau d'activité au plus près de son potentiel, n'est pas guidée par des indicateurs rendus obsolètes par les politiques structurelles. En définitive, il n'y a là rien de nouveau: un des défis permanents de la politique macroéconomique est de s'appuyer sur des indicateurs aussi fiables que possible.

\section{La monnaie commune: un problème supplémentaire?}

La politique monétaire est l'un des instruments de la politique macroéconomique. La monnaie commune implique que cet instrument n'est plus disponible au niveau national. Cela signifiet-il que la conclusion qui précède - la politique macroéconomique doit continuer à jouer son rôle habituel - devient inapplicable? Il est vrai que la politique monétaire est l'instrument macroéconomique le plus souple et que son transfert au niveau de la zone euro peut poser problème. La Banque centrale européenne (BCE), en effet, ne peut pas, et ne doit pas, ajuster sa politique aux besoins particuliers de tel ou tel de ses membres. 
Il reste cependant à ne pas surestimer la perte de l'instrument de politique monétaire. L'exemple du passage à l'euro est instructif. La figure 2 montre ce qui s'est passé dans deux pays au moment où ils ont rejoint la zone euro : le Portugal en 1999 et la Grèce en 2001. Le Portugal a alors connu une hausse du taux de chômage. Or la politique monétaire y est devenue fortement expansionniste comme l'indique la baisse spectaculaire du taux d'intérêt réel. L'explication est que la politique budgétaire est devenue très contractionniste, comme l'indique l'augmentation du solde budgétaire corrigé des fluctuations cycliques (mesuré sur l'échelle de gauche). La montée du chômage après l'adoption de l'euro n'est donc pas une conséquence de ce changement, mais d'un choix de politique budgétaire - qui pouvait être motivé par la montée de la dette publique. En Grèce, par contre, le chômage a connu une pente descendante. Durant la période 2001-2004, les deux instruments de politique macroéconomique ont contribué à supporter la croissance. Ces exemples suggèrent qu'une utilisation judicieuse de la politique budgétaire permet de contrebalancer la perte de l'instrument monétaire. introduits en 2005. Une interprétation est que le pacte est devenu suffisamment flexible pour permettre à chaque pays d'utiliser son instrument de politique macroéconomique en fonction de ses besoins. Une autre interprétation est que les ambigüités qui caractérisent le nouveau pacte sont de nature à compliquer la tâche des autorités nationales lorsqu'elles conduisent des réformes structurelles. Alors qu'il sera difficile de bien mesurer le potentiel de croissance, en raison des changements structurels, il est probable que des divergences d'opinion apparaîtront sur ce point entre un gouvernement soucieux de se ménager des marges de manœuvre et la Commission européenne par nature prudente.

\section{Les conditions politico- économiques des réformes}

Un autre aspect de l'utilisation de la politique macroéconomique concerne les aspects politiques de la mise en œuvre de réformes de structure. Une opinion largement partagée est que les réformes

Figure 2: Taux de chômage, taux d'intérêt réel et solde budgétaire (en \% du PIB) au Portugal et en Grèce (1995-2005)
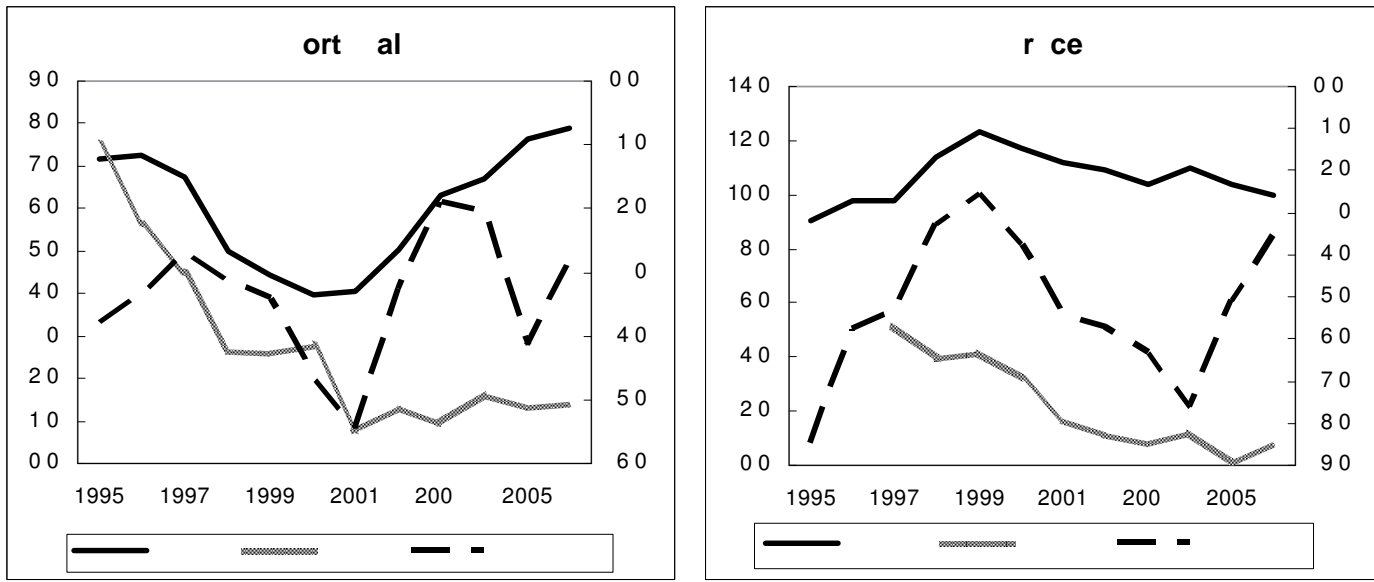

Source: Perspectives économiques, OCDE, 2006.

Note: le taux d'intérêt réel est calculé ex post comme la différence entre le taux d'intérêt long nominal et le taux d'inflation annuel.

Une première conclusion est que c'est la politique budgétaire qui devra assurer l'essentiel du rôle stabilisateur de la politique macroéconomique. Certes, l'instrument est plus grossier que la politique monétaire et son utilisation est soumise à des aléas politiques dont une banque centrale indépendante est protégée. L'implication est qu'il serait désirable d'améliorer les conditions de mise en œuvre de la politique budgétaire. Des propositions en ce sens sont formulées dans WyPLOSZ (2005).

Une deuxième conclusion est que le pacte de stabilité et de croissance ne doit pas bloquer l'utilisation de la politique budgétaire. Il est trop tôt pour savoir comment fonctionnera le pacte après les changements sont plus facilement acceptées en période de croissance rapide et de chômage déclinant que lorsque les conditions sont difficiles. Plusieurs arguments sont avancés en ce sens.

\section{Aider la mise en route des réformes}

En premier lieu, il n'est pas exclu que l'impact immédiat des réformes structurelles soit de ralentir la croissance. La remise en cause d'arrangements en matière de conditions de travail, de recrutement et de licenciement, par exemple, est une source d'incertitude qui peut affecter négativement la consommation et l'investissement des entreprises. La crainte de créer une telle situation, et de provo- 
quer ainsi une hostilité aux réformes, est de nature à décourager le gouvernement d'entreprendre des réformes. Une croissance vigoureuse, temporairement stimulée par une politique macroéconomique expansionniste, est donc souhaitable, à la fois pour rassurer le gouvernement et pour atténuer, voire éliminer l'impact contractionniste des réformes.

Un autre argument concerne les transferts parétiens. En général, une réforme remet en cause des positions établies. Si la réforme est bien conçue, les bénéfices qu'en retire la société excèdent largement les coûts subis par certains - la réforme est dominante au sens de Pareto. Dans ces conditions, le gouvernement peut trouver équitable, et politiquement utile, de compenser ceux qui pâtissent des réformes au moyen de transferts. Ces transferts alourdissent les dépenses. En période de croissance rapide, cependant, les revenus fiscaux augmentent et peuvent permettre au gouvernement de financer tout ou partie des transferts.

Finalement, de nombreux aspects des réformes contenues dans la Stratégie pour l'emploi de l'OCDE impliquent des sacrifices de la part des employés et des chômeurs. Ces sacrifices sont toujours impopulaires, ils le sont encore plus en période de croissance faible quand le chômage augmente. D'où l'idée d'utiliser la politique macroéconomique pour créer une situation de croissance dynamique au moment de la mise en œuvre des réformes.

\section{Les limites du soutien politique de la politique macroéconomique}

Face à ces arguments en faveur d'un usage stratégique de la politique macroéconomique pour améliorer l'acceptabilité politique des réformes, d'autres arguments vont dans le sens opposé. Ainsi, on peut considérer que c'est lorsque la situation macroéconomique est difficile que les divers groupes de pression qui s'opposent politiquement aux réformes sont le plus mal placés pour défendre le statu quo(1). Une réforme consiste, en effet, à modifier le statu quo. Même s'ils comprennent que la réforme est globalement bénéfique, tous ceux qui ont quelque chose à y perdre ont naturellement tendance à reporter sur d'autres le coût de la réforme. Le résultat est un blocage total, chaque groupe de pression espérant que les autres concéderont avant lui. La situation perdure aussi longtemps que le coût du statu quo est moins élevé que le coût de concéder. Une politique macroéconomique expansionniste est de nature à réduire le coût du statu quo et risque donc de repousser le moment où la réforme devient politiquement acceptable.

Le raisonnement qui précède part de l'idée que les groupes de pression, et donc l'opinion publique,

(1) La théorie sous-jacente à cet argument est développée par Alesina et Drazen (1991). comprennent que les réformes sont globalement bénéfiques. En pratique, rien n'est moins sûr. Tant que la situation macroéconomique (croissance, emploi) est acceptable, il est souvent difficile de convaincre l'opinion publique du besoin de modifier le statu quo. Ceci signifie qu'une politique macroéconomique neutre, qui cesse de doper la croissance, peut aider à la réalisation que la situation est en fait détériorée et qu'un remède sérieux est nécessaire. Autrement dit, une politique macroéconomique expansionniste risque d'effacer la "preuve» que des réformes structurelles sont indispensables.

Une version plus radicale de cette argumentation est que «les chants les plus désespérés sont les chants les plus beaux». C'est lorsque la situation est fort dégradée que le gouvernement peut le mieux obtenir le soutien politique nécessaire pour s'attaquer aux blocages de l'économie. Le cas d'école est celui de la Grande-Bretagne. Après deux décennies de croissance insuffisante, et ce malgré des politiques macroéconomiques qui ont conduit à une inflation élevée et une dette en augmentation, Margaret Thatcher a remporté les élections sur le thème TINA (there is no alternative): il n'y a pas d'autre voie que celles des réformes de structure. Il existe d'autres versions de cet argument (la parabole du dos au mur est aussi fréquemment évoquée) qui combine les deux précédents: une situation macroéconomique dégradée révèle la gravité de la situation aux yeux d'une opinion publique naturellement sceptique et, en même temps, rend le coût du statu quo excessif(2).

\section{Les leçons des expériences}

Le débat qui précède n'est pas nouveau. De nombreux travaux ont cherché à déterminer quelles sont les conditions macroéconomiques qui tendent à rendre politiquement acceptables des réformes de structure. Une récente synthèse produite par le Fonds monétaire international (2004) examine l'expérience accumulée dans les pays avancés de 1982 à 1998. Cette étude distingue divers domaines de réformes (marché du travail, marché des biens et services, fiscalité, commerce international et secteur financier). Dans tous les cas sauf celui du marché du travail, les réformes tendent à être mises en œuvre durant les années de faible croissance ou juste après, durant la phase de reprise économique. L'exception est le marché du travail où, semble-t-il, les réformes ont plutôt lieu durant les années de croissance rapide.

Ces résultats indiquent que les différents arguments présentés précédemment sont, à des degrés divers, validés, même s'ils sont en apparence contradictoires. Des crises sérieuses peuvent favoriser des

(2) Une version extrême est celle de la terre brûlée ou de la politique du pire; bien sûr, nul ne défend une telle vue. 
réformes, mais il est difficile de réformer le marché du travail quand l'économie est en basse conjoncture. Les sorties de récession sont un moment favorable pour presque tous les types de réformes, probablement parce que les réformes sont préparées et décidées durant la période déclinante du cycle et, lorsqu'elles sont mises en place, la reprise est en cours.

Au total, donc, l'argument selon lequel une politique macroéconomique expansionniste doit être utilisée comme préalable aux réformes ne semble pas validé, sauf en ce qui concerne les réformes du marché du travail. Il est probablement peu judicieux de chercher à «manipuler» les conditions macro économiques pour créer des conditions favorables aux réformes structurelles.

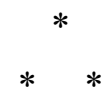

Les réformes structurelles et la politique macroéconomique ont peu de points en commun. Les premières doivent être conduites indépendamment des secondes, dont l'objet est le lissage des cycles conjoncturels. Les quelques points de contact sont les suivants.

Dans la mesure où les réformes structurelles ont des effets cycliques, la politique macroéconomique peut devoir être ajustée en conséquence. Il ne s'agit pas, cependant, d'un rôle additionnel pour la politique macroéconomique.

Dans son rôle traditionnel, la politique macroéconomique a pour objectif de maintenir l'activité (PIB, emploi, chômage) proche de son niveau potentiel. Les réformes structurelles ont pour objectif de changer ces différents points de références ainsi que leurs interrelations. Elles ouvrent donc une période de plus grande incertitude pour la politique macroéconomique dont les références, habituellement déjà établies avec peu de précision, deviennent plus imprécises encore. Durant cette période d'apprentissage, les responsables de la politique macroéconomique doivent donc être encore plus vigilants que d'habitude. Des erreurs sont probables, c'est là un des coûts indirects des réformes structurelles.

La principale difficulté des réformes structurelles est leur acceptabilité politique. Globalement bénéfiques - dominantes au sens de Pareto - les réformes imposent des coûts à certains groupes qui, naturellement, s'y opposent. Un débat oppose ceux qui pensent que la politique macroéconomique se doit de créer des conditions politiquement favorables aux réformes et ceux qui considèrent que les réformes sont d'autant plus probables que la situation est difficile et reconnue comme telle pour des raisons structurelles, et non conjoncturelles. Les leçons des expériences passées confirment ces deux points de vue qui ne sont pas nécessairement incompatibles entre eux. Plus précisément, les réformes du marché du travail tendent à être adoptées durant des périodes de conjoncture favorable, alors que des conditions macroéconomie défavorables créent les conditions qui rendent acceptables les autres réformes.

L'appartenance de la France à la zone euro affecte la conduite de la politique macroéconomique. Privée de l'instrument de la politique monétaire, la France, comme les autres pays membres, doit faire un usage plus intensif de la politique budgétaire. Les contraintes du pacte de stabilité et de croissance peuvent être contre-productives. Étant donné que le pacte a été récemment remanié, il n'est pas clair, à ce stade, comment ces contraintes vont opérer.

\section{Bibliographie}

Alesina A., Drazen A. (1991), "Why Are Stabilizations Delayed?", American Economic Review 81 : 1170-88.

Blanchard O., Summers L. (1986), "Hysteresis and the European Unemployment Problem”, NBER Macroeconomics Annual 1, 15-78.

Gustavsson M., Österholm P. (2006), “Does Unemployment Hysteresis Equal Employment Hysteresis ?", Working Paper 2006: 15, Department of economy, d'Uppsala University.

Høu J., Galasso V., Nicoletti G., Dang Th.Тн. (2006), "The Political Economy of Structural Reform: Empirical
Evidence from OECD Countries", Economics Department, Working Paper, OCDE.

INTERNATIONAL MONETARY FOUND (2004), World Economics Outlook, April, Chapter 3.

Ocde (2006), Perspectives de l'emploi de l'OCDE. Stimuler l'emploi et les revenus, disponible sur le site: http:// www.sourceocde.org/emploi/9264023860

Wyplosz C. (2005), "Fiscal Policy: Institutions Versus Rules”, National Institute Economic Review 191 : 70-84. 